\title{
RESONANT STELLAR ORBITS \\ IN SPIRAL GALAXIES
}

\author{
P. O. VANDER VOORT \\ University of Chicago, Chicago, Ill., U.S.A.
}

\begin{abstract}
This paper reviews a series of investigations of the orbits of stars in the regions of the Lindblad resonances of a spiral galaxy. The analysis is formulated in an epicyclic approximation. Analytic solutions of the epicyclic equations of motion are obtained by the method of harmonic balance of Bogoliubov and Mitropolsky. These solutions represent the resonance phenomena exhibited by the orbits in generally excellent agreement with numerical solutions.
\end{abstract}

\section{Introduction}

In recent years, it has become apparent that the clarification of important aspects of the dynamics of spiral structure in galaxies would require a systematic study of stellar orbits in the regions of the so-called Lindblad resonances. In a series of investigations (Vandervoort, 1973, 1975; Vandervoort and Monet, 1975), it has been possible to formulate an analytic theory of these orbits in an epicyclic approximation, to test this theory with the aid of numerical solutions of the epicyclic equations of motion, and to make use of these analytic and numerical studies in order to survey the resonance phenomena exhibited by the orbits. Apart from its applications to problems of spiral structure, the work is of interest, because it deals with a relatively simple dynamical system which exemplifies important features of the study of resonant orbits in stellar dynamics.

\section{The Epicyclic Theory of the Orbits}

Specifically, we consider the orbit of a star in the plane of a galaxy in which the prevailing gravitational potential is a superposition of a dominant axisymmetric component and a small non-axisymmetric perturbation of the form

$$
\begin{aligned}
\mathfrak{B}(\varpi, \theta) & =\mathfrak{B}^{(a)}(\varpi)+\operatorname{Re}\left[\mathfrak{B}^{(1)}(\varpi) \exp (\operatorname{im} \theta)\right] \\
& =\mathfrak{V}^{(a)}(\varpi)+\mathfrak{B}^{(1)}(\varpi, \theta), \quad(\text { say }),
\end{aligned}
$$

where $m$ is an integer. Here $\varpi$ and $\theta$ are the radial and azimuthal coordinates of the star, respectively, in a frame of reference rotating uniformly with angular velocity $\Omega$. The canonical momenta conjugate to these coordinates are the $\varpi$-component of the velocity $\Pi$ and the angular momentum $h$, respectively. We introduce epicyclic variables $\varpi_{1}, \theta_{1}, \Pi_{1}$, and $h_{1}$, by referring the motion of the star to a circular orbit in the potential $\mathfrak{B}^{(a)}(\varpi)$. Let the circular orbit have a radius $\varpi_{0}$ and an initial azimuthal coordinate $\theta_{0}$, and let $\Omega_{0}-\Omega$ denote its angular velocity (in the rotating frame) and $h_{0}$ 
its angular momentum. The epicyclic variables are defined by the relations

$$
\begin{aligned}
& \varpi=\varpi_{0}+\varpi_{1}, \quad \theta=\theta_{0}+\left(\Omega_{0}-\Omega\right) t+\theta_{1}, \\
& \Pi=\Pi_{1}, \quad \text { and } \quad h=h_{0}+h_{1},
\end{aligned}
$$

where $t$ denotes the time.

In the epicyclic approximation, the equations of motion happen to be the canonical equations associated with the Hamiltonian

$$
H_{1}=\frac{1}{2} \Pi_{1}^{2}+\frac{1}{2} \omega^{2} \varpi_{1}^{2}-\frac{2 \Omega_{0}}{\varpi_{0}} \varpi_{1} h_{1}+\frac{h_{1}^{2}}{2 \varpi_{0}^{2}}+\mathfrak{B}^{(1)}(\varpi, \theta),
$$

where $\omega$ is the epicyclic frequency, the arguments of $\mathfrak{B}^{(1)}$ are interpreted in accordance with Equations (2) and $\Pi_{1}$ and $h_{1}$ are the canonical momenta conjugate to the coordinates $\varpi_{1}$ and $\theta_{1}$, respectively. The quantity

$$
H_{E}=H_{1}+\left(\Omega_{0}-\Omega\right) h_{1}
$$

is an exact integral of these equations of motion, and this integral expresses (in the epicyclic approximation) the constancy of Jacobi's integral. The Hamiltonian character of the epicyclic equations and the existence of an exact integral of those equations which is both approximate and analogous to Jacobi's integral ensure that the epicyclic theory will provide a good model of the orbits.

For the sake of definiteness, we shall concentrate on the orbits in the region of the inner Lindblad resonance where the quantity

$$
v=\frac{m\left(\Omega_{0}-\Omega\right)}{\omega}
$$

has the value +1 . A similar treatment applies to the region of the outer resonance where $v=-1$.

The epicyclic equations are solved by the method of harmonic balance (Bogoliubov and Mitropolsky, 1961). This is a perturbation theory in the amplitude of $\mathfrak{B}^{(1)}(\varpi, \theta)$. The essence of the method is the manner in which it allows for large perturbations of the amplitudes and phases of the epicyclic motion by the resonance. The solution is written in the form

$$
\begin{aligned}
& \varpi_{1}=\varpi_{10}(t) \sin [v \omega t+\varphi(t)]+\frac{2 \Omega_{0}}{\omega^{2} \varpi_{0}} h_{10}(t)+\cdots, \\
& \theta_{1}=\frac{2 \Omega_{0}}{\omega \varpi_{0}} \varpi_{10}(t) \cos [v \omega t+\varphi(t)]+\theta_{10}(t)+\cdots,
\end{aligned}
$$

and

$$
h_{1}=h_{10}(t)+\cdots,
$$

where we are showing only the dominant terms, and we are omitting the solution for $\Pi_{1}$ as inessential to the present discussion. The functions $\varpi_{10}(t), \varphi(t), h_{10}(t)$, and $\theta_{10}(t)$, satisfy certain differential equations which are determined by a requirement 
that the time-dependence of these functions gives rise to terms in the equations of motion which just balance the resonant parts of the perturbing forces. This procedure eliminates terms with small denominators and secular terms which appear in solutions obtained with the aid of more conventional perturbation theories.

With the solutions which are obtained for $\varpi_{10}(t), \varphi(t), h_{10}(t)$, and $\theta_{10}(t)$, Equations (2), (6), (7), and (8), reproduce, at least qualitatively, all of the resonance phenomena which Contopoulos (1970) has found in numerical solutions for the orbits. (1) There are solutions in which $\varpi_{10}, h_{10}$, and the linear combination $m \theta_{10}+\varphi$, are all constants. In this case the circular and epicyclic motions are commensurate, and the orbits are periodic. Both stable and unstable periodic orbits are represented. (2) There are solutions in which $\varpi_{10}, h_{10}$, and $m \theta_{10}+\varphi$, are all periodic functions of time. These solutions represent tube orbits which oscillate around stable periodic orbits. The circular and epicyclic motions are commensurate only in the sense of a longtime average. (3) Finally, there are solutions in which $\varpi_{10}$ and $h_{10}$ are periodic functions of time whereas $m \theta_{10}+\varphi$ is a superposition of periodic and linear functions of time. In this case, the circular and epicyclic motions are not commensurate even in the sense of a long-time average. These solutions represent non-resonant orbits.

The solution of the epicyclic equations along these lines leads to the construction of a formal, isolating integral of the motion in addition to the integral of the Jacobi type given in Equation (4). Near the inner Lindblad resonance, the new integral is of the form

$$
I=h_{10}+\frac{1}{2} m \omega \varpi_{10}^{2}+\cdots
$$

through its dominant terms.

\section{The Comparison of Analytic and Numerical Solutions of the Epicyclic Equations of Motion}

We have compared the analytic solutions of the epicyclic equations with numerical solutions in case

$$
\mathfrak{B}^{(1)}(\varpi)=A \exp (i k \varpi)
$$

in the perturbation in Equation (1), where the amplitude $A$ and the wavenumber $k$ are complex constants. This choice of the form of the perturbation reduces the Hamiltonian given in Equation (3) to a well-defined model problem suitable for systematic numerical tests of the analytic theory. Moreover, it is of a form which is appropriate, at least locally, for a tightly-wound spiral pattern. For the latter reason, the calculations performed in the comparison of analytic and numerical solutions also provide a useful survey of the resonance phenomena.

It is actually the surfaces of section derived from corresponding families of analytic and numerical solutions which have been compared. In the case at hand, a surface of section is essentially the $(\varpi, \Pi)$-plane for a fixed value of Jacobi's integral (Contopoulos, 1970). A given orbit is represented in the surface of section by points 
defined by the pairs of values $(\varpi, \Pi)$ which occur when the azimuthal coordinate $\theta$ returns to a specified value $\theta_{i}$, say. If the orbit is one for which the equations of motion admit a second isolating integral, in addition to Jacobi's integral, then these points lie on a simple closed contour called an invariant curve.

In Figure 1, we compare the analytic and numerical versions of a particular surface of section. This example is representative of the situations which might occur in the Galaxy. The important parameters of the problem have been assigned the values $\Omega_{0}=0.65, m=2, v=0.93$, and $k=20-2 i$, where we have adopted $\varpi_{0}$ as the unit of length and $\omega^{-1}$ as the unit of time. The value of $A$ is chosen so that the amplitude of the spiral component of the field is $5 \%$ of the strength of the axisymmetric component. The choice of the values of $\varpi_{0}$ and Jacobi's integral is such that $H_{E}=0$ (see Equation (4)).

The main features of this surface of section are three stable periodic orbits, their associated families of tube orbits, and two unstable periodic orbits. The analytic and numerical versions agree very well in the representation of these features. Solid contours represent well-defined invariant curves in both versions. All orbits have welldefined invariant curves in the analytic theory in virtue of the existence of the second isolating integral. While most orbits are found numerically to have well-defined invariant curves, a few do not. In the numerical version of the surface of section, orbits without invariant curves are represented schematically by dashed contours. The first three dashed contours, as we count outward from the central periodic orbit, represent orbits which form islands in the surface of section. One such system of island, corresponding to the innermost dashed contour in Figure 1, is shown in Figure 2. The remaining two dashed curves in Figure 1 represent invariant curves which have dissolved as a consequence of their proximity to the unstable periodic orbit which lies between them. The two dissolved invariant curves are shown in Figures 3 and 4 .
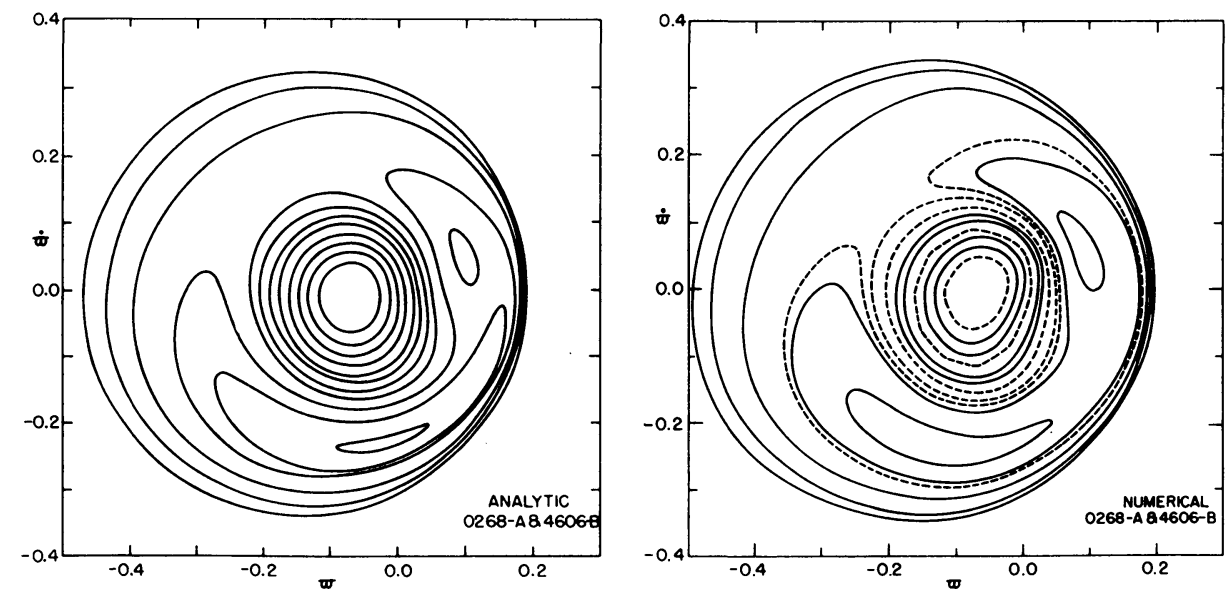

Fig. 1. The surface of section described in the text as derived from analytic and numerical solutions of the epicyclic equations. Note that in this and the following figures the $\varpi$-axis has been labeled with the values of $\varpi-\varpi_{0}(=\varpi-1$ in the adopted system of units). 


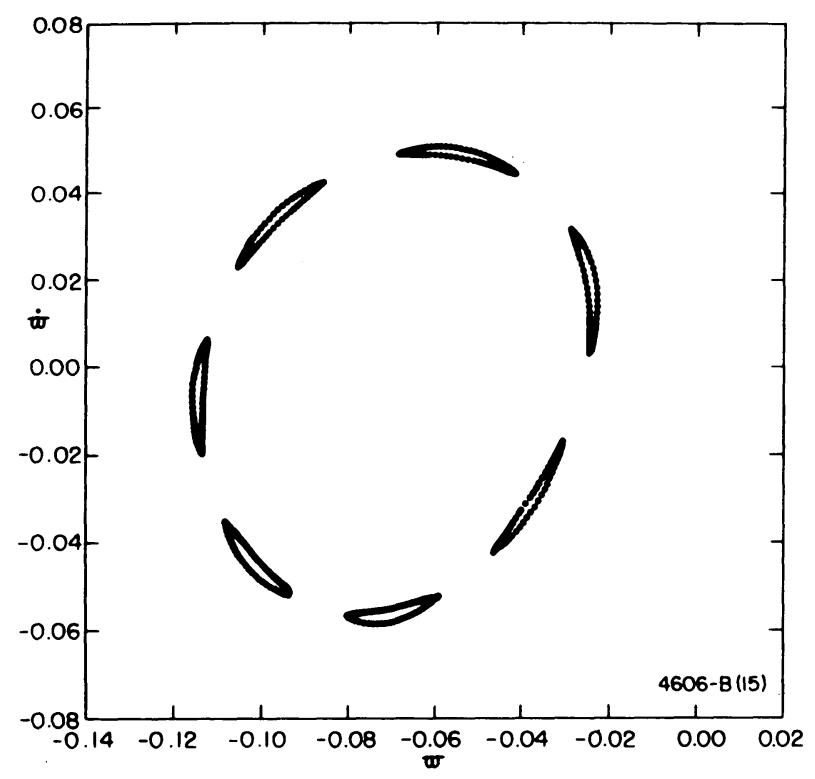

Fig. 2. An example of a system of islands.

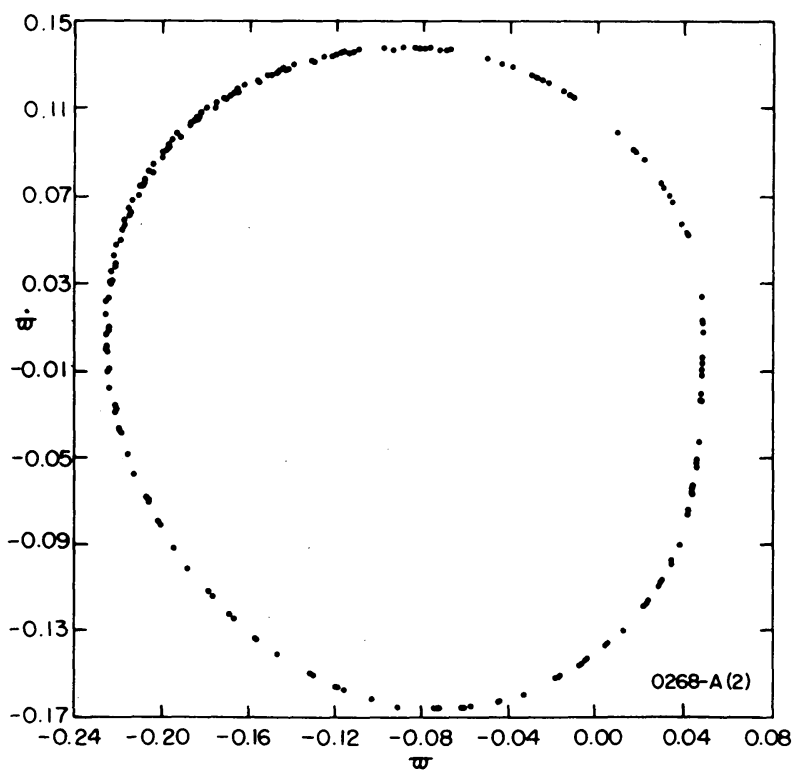

Fig. 3. An example of the dissolution of an invariant curve. 


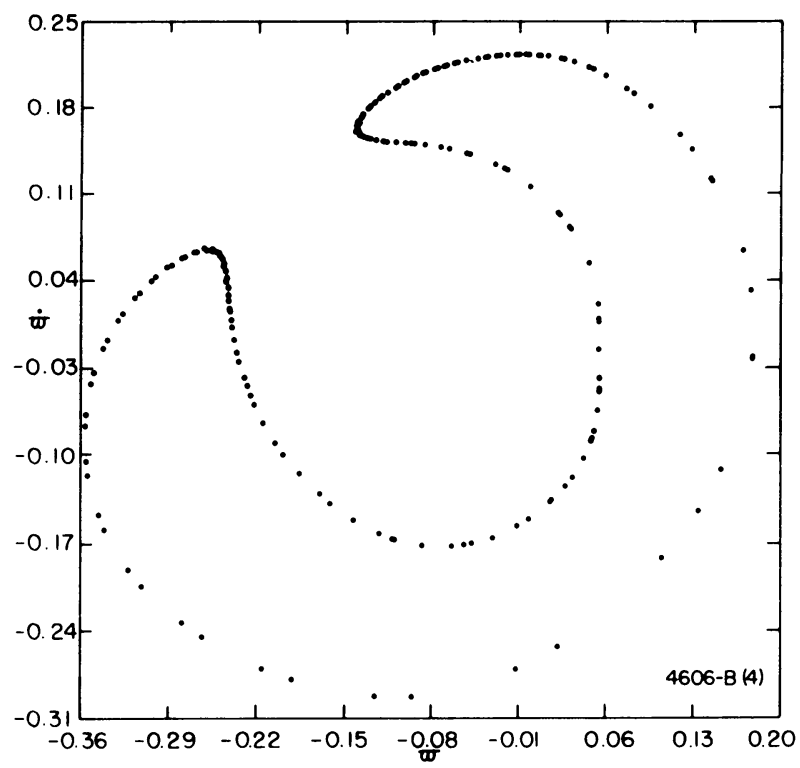

Fig. 4. An example of the dissolution of an invariant curve.

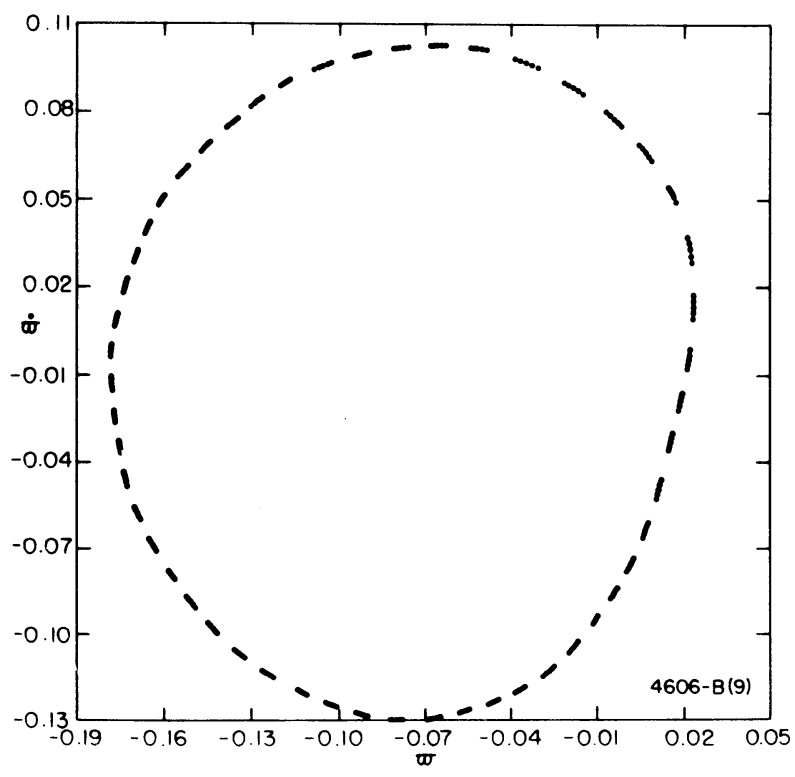

Fig 5. An example of a well-defined invariant curve. 
Finally, we show in Figure 5 the points in the surface of section representing an orbit which is judged to have a well-defined invariant curve.

The results described above are typical of what have been obtained under a wide range of conditions. However, two points should be noted. First, it is more commonly the case that a surface of section will contain only two stable periodic orbits, their associated families of tube orbits, and only one unstable periodic orbit. Secondly, when we reduce the amplitude of the perturbation by a factor 10 , as may be appropriate if the spiral structure is damped in the resonance region, the numerical results show no detectable tendency for the dissolution of invariant curves or for the formation of islands.

\section{Concluding Remarks}

(1) The solutions of the epicyclic equations obtained by the method of harmonic balance account for the main resonance phenomena in a simple and natural manner. The agreement of analytic and numerical solutions is very good, especially when one considers that the analytic solutions used in these comparisons were truncated at the lowest order of approximation.

(2) The construction of two isolating integrals of the motion is an important aspect of the theory. It is in terms of such integrals that one would hope to incorporate the resonance phenomena into the dynamics of spiral structure.

(3) The tendency for the dissolution of invariant curves and for the formation of islands is more fully developed than might have been expected for the perturbations considered. However, these phenomena do not appear to be so fully developed that they would vitiate applications of the analytic theory to the dynamics of spiral structure.

(4) The qualitative agreement of the present results, all obtained at the level of the epicyclic approximation, with the properties of numerical solutions of the exact equations of motion (Contopoulos, 1970) confirm the validity of the epicyclic orbits as models of the exact orbits. However, more quantitative comparisons are of practical interest, and they will be made.

\section{Acknowledgements}

The numerical parts of these investigations have been done in collaboration with $\mathrm{Mr}$ David G. Monet, who deserves the highest praise for the effort, initiative, and enthusiasm, which he has brought to the work. Our research has been supported in part by the National Science Foundation through Grant GP-17639 to the University of Chicago.

\section{References}

Bogoliubov. N. N. and Mitropolsky, Y. A.: 1961, Asymptotic Methods in the Theory of Non-Linear Oscillations, Gordon and Breach, New York, $\$ 14$.

Contopoulos, G. : 1970, Astrophys. J. 160, 113.

Vandervoort. P. O.: 1973. Astrophys. J. 180, 739 
Vandervoort, P. O.: 1975, Astrophys. J. (to be submitted).

Vandervoort, P. O. and Monet, D. G. : 1975, Astrophys. J. (to be submitted).

\section{DISCUSSION}

Contopoulos: I was glad to check that Dr Vandervoort's results about the new integral near the inner Lindblad resonance agree with mine (Astrophys. J. 160, 113, 1970). Perhaps the most simple way to describe these resonance phenomena is by using action-angle variables (introduced in spiral structure theory by Kalnajs and Lynden-Bell). In these variables the Hamiltonian takes the form

$$
H=\omega_{1} I_{1}+\omega_{2} I_{2}+f_{21}\left(I_{1}, I_{2}\right) \cos \left(\theta_{1}-2 \theta_{2}\right)+\text { higher order terms. }
$$

Near the resonance we can use canonical changes of variables to eliminate all trigonometric terms except those containing the combination $\left(\theta_{1}-2 \theta_{2}\right)$. Thus we find a resonant integrable case of the type discussed earlier this morning.

It is easy to see that the combination $J_{2}=I_{2}+2 I_{1}$ is an integral of motion. This is the appropriate adiabatic invariant near resonance, while $I_{1}$ and $I_{2}$ separately are not.

I will discuss this problem further at the Paris Meeting next week.

Vandervoort: The problem is one in two degrees of freedom. Therefore, the epicyclic motion is described in terms of two amplitudes and two phases. However, the construction of the second integral involves only a linear combination of the phases, and this is precisely your linear combination of angle variables.

Lynden-Bell: I would like to point out the physical reason for the constant of the motion at resonances. If one looks in the axes rotating with the spiral wave the resonant orbits exactly close and the near resonant orbits move slowly. The circulation around the orbits is rapid compared with the slow movement of the near resonant orbit so the action corresponding to that circulation is adiabatically invariant. This adiabatic invariant is the constant of the motion. Born in his book The Mechanics of the Atom shows a beautiful method of treating all these problems in Angle and Action variables and combining them into slow and fast changing variables near resonances. This is the best way of treating this problem.

Contopoulos: I would only like to add that the appropriate adiabatic invariant is a different combination of $I_{1}$ and $I_{2}$ at each resonance.

Colin: Have you made some identical work for corotation resonance?

Vandervoort: No, I have not. I suspect that the method might encounter difficulties near the corotation point. 